\title{
Proximidad, territorio e innovación. Una aproximación crítica desde categorías ausentes: escalaridad, estructuración social y periferia
}

\section{Proximity, territory and innovation. A critical approach from absent categories: scalarity, social structuring and periphery}

\author{
Ignacio Trucco' (1) y Victor Ramiro Fernandez² (1)
}

\begin{abstract}
RESUMEN
Desde mediados de la década de 1980 hasta la actualidad, diversas intervenciones pusieron en foco las relaciones entre proximidad, territorio e innovación. El debate se estructuró en torno a dos preguntas subyacentes: ¿qué liga a la sociedad con el espacio geográfico?, y ¿cuáles son las razones que definen su desempeño económico? En este trabajo se ofrece una interpretación del debate, mostrando las principales hipótesis en juego, las limitaciones que enfrentaron y las líneas de investigación que se abren a partir de allí. Se argumentará que las ideas en pugna oscilaron entre el enfoque marginalista y el relacional y que la creciente importancia y autonomía asignada a las instituciones favoreció el predominio del segundo sobre el primero. Se observa también que los fundamentos relacionales, al precisarse y ganar centralidad, pusieron en evidencia sus principales limitaciones. Las mismas se expresaron, primero, en una desconexión estructural entre la sociedad y el espacio geográfico, que luego se extiende a la incapacidad de definir las bases del desempeño económico relativo, la escalaridad de los sistemas de producción, sus divisiones sociales internas y la estructuración centro-periferia del sistema mundial. El artículo concluye con un balance de la agenda de investigaciones en base a las apreciaciones realizadas.
\end{abstract}

Palabras clave: sistemas productivos locales, espacio relacional, espacio social, producción del espacio, histórico-estructural.

\section{ABSTRACT}

From the mid-1980s to the present day, there has been a long debate focused on the relationships between proximity, territory and innovation. This debate was structured around two underlying questions: what links society with geographic space?; and what are the reasons that affect its economic performance? This work offers an interpretation of the debate, showing the main hypotheses at stake, the limitations they faced, and the lines of research that open up from there. It is argued that the competing ideas oscillated

Instituto de Humanidades y Ciencias Sociales del Litoral - Universidad Nacional del Litoral - Consejo Nacional de Investigaciones Científicas y Técnicas. Correo electrónico: ignacio.trucco@gmail.com

Instituto de Humanidades y Ciencias Sociales del Litoral - Universidad Nacional del Litoral - Consejo Nacional de Investigaciones Científicas y Técnicas. Correo electrónico: victorramirofernandez@hotmail.com 


\begin{abstract}
between the marginalist and the relational approach and the growing importance and autonomy assigned to the institutions that favor the predominance of the second over the first. It is also observed that the relational foundations, by specifying and gaining centrality, revealed their main limitations. They are expressed in a structural disconnection between society and geographic space, which then extends to the inability to define the bases of relative economic performance, the scale of production systems, their internal social divisions and the center-periphery structuring of world system. The article concludes with a balance of the research agenda based on the assessment made.
\end{abstract}

Keywords: local productive systems, relational space, social space, production of space, historical-structural.

El vínculo entre diferenciación social y la diferenciación geográfica, constituye uno de los problemas, más importantes de la geografía humana, y un punto de encuentro con la teoría social y económica.

Uno de los capítulos de esta extensa problemática puso su foco sobre las relaciones entre proximidad, territorio e innovación y, desde mediados de la década de 1980 hasta la actualidad, estos conceptos fueron estudiados a partir de dos interrogantes básicos: ¿qué liga a la sociedad con el espacio geográfico?, y ¿cuáles son las razones que definen el desempeño económico de los distintos espacios? El trabajo se propone ofrecer una interpretación de dicho debate, mostrando las principales hipótesis en juego, las limitaciones que enfrentaron los enfoques intervinientes y las líneas de investigación que, a partir éstas, podrían abrirse.

El primer argumento constituye una hipótesis de lectura según la cual las ideas en pugna definieron un clivaje entre los supuestos básicos de dos teorías socioeconómicas: el marginalismo y el enfoque relacional. Se intenta argumentar que, más allá de evidentes mixturas teóricas y disciplinares, desde el primer momento, se trazó una distinción entre el fundamento utilitarista y el modelo relacional de la acción social. En segundo lugar, se intentará mostrar cómo, en el desarrollo del debate mismo, se acentuó la observación de la importancia y autonomía de las instituciones no mercantiles, lo que favoreció el predominio de las ideas relacionales en detrimento de los fundamentos marginalistas. Sin embargo, ello hizo que el enfoque relacional deba explicitar sus propios fundamentos exponiendo allí algunas limitaciones.

El trabajo establece una interpretación de dichas limitaciones sobre la base de las siguientes ideas: 1) El modelo relacional conduce a una separación estructural entre el espacio social y el espacio geográfico que no puede ser superada. 2) Como consecuencia de lo anterior, el desempeño económico recibe una interpretación arbitraria, apoyada en realidades institucionales ad hoc. 3) Finalmente, esto tiene como consecuencia, que los modos de diferenciación o estructuración socio espacial se tornan ininteligibles y no pueden ser definidas sus articulaciones. En particular, se consideran tres categorías ausentes que definen a los sistemas de producción: la estructuración multi escalar, la estructuración social y la estructuración mundial con espacios centrales y periféricos.

El manuscrito repasa el desarrollo del debate a partir de las principales intervenciones realizadas, y se intenta mostrar, a la par, cómo la metáfora con la que el enfoque relacional se refiere a la realidad social misma resulta la base de las limitaciones arriba descriptas. Esta metáfora es la red de relaciones intersubjetivas, cuya definición es independiente del contenido de dichas rela- 
ciones. Se trata, por lo tanto, de una realidad relacional genérica o abstracta, sin atributos histórico-estructurales específicos, que establece el molde o el lenguaje de lo que la teoría capta como realidad socio-espacial que, luego, es decir, a posteriori, es dispuesta sobre el espacio geográfico.

El trabajo concluye planteando que, para poder captar los modos de diferenciación o estructuración socio espacial, los sistemas productivos, deberían ser abordados como el producto de relaciones sociales de producción en su especificidad histórico-estructural. En el modo que en ellas despliegan y articulan entre sí, podrían hallarse las claves de una lectura integral, que dé inteligibilidad simultánea, a la estructuración escalar, social y geográfica, y a las razones del desempeño relativo de los mismos.

\section{Proximidad e innovación en la formulación californiana}

Las relaciones entre proximidad, territorio e innovación encuentran su antecedente principal en el debate regional que se desarrolló a mediados de la década de 1980, mientras el capitalismo mundial giraba hacia la globalización financiera de acelerada mercantilización. Incluso este debate se apoyó en una reevaluación más general que, en esta misma época, se produjo sobre la relación entre la sociedad y el espacio que involucró diferentes perspectivas.

Derek Gregory y John Urry editaron en 1985 un conocido trabajo en donde se incorporan algunas de las principales voces al respecto y se establece la problemática que está en la base de las investigaciones aquí analizadas: cómo conceptualizar, simultáneamente, la estructuración social, en tanto espacio de relaciones, y la base material, es decir, el espacio de estas relaciones (Gregory \& Urry, 1985). Gregory y Urry identifican en la introducción una idea clave para este trabajo: el determinismo geográfico es, simplemente, una imposibilidad (Gregory \& Urry, 1985: 3). Aún en la más reductiva de las perspectivas sobre la estructura espacial, intervienen ideas que remiten a relaciones sociales que condicionan o incluso producen la dicha estructuración. Sin embargo, clarificar los modos en que ambos momentos se conectan no es algo trivial e incluso constituye el plafón en donde se definen las relaciones entre proximidad, territorio e innovación en la literatura especializada.

Esto puede verse en una primera caracterización del debate realizada por Michael Storper (1995) quien distinguió un primer conjunto de intervenciones que pusieron énfasis en la "ruptura industrial" (Piore \& Sabel, 1986) basada en las tecnologías de la información y la comunicación (control numérico) y las nuevas formas institucionales de regulación del capitalismo post fordista.

Esta literatura abonó un imaginario económico según el cual los sistemas regionales de pequeñas y medianas empresas, basados en la competencia y la cooperación, determinan la gobernanza más eficiente en la era del post fordismo y la especialización flexible (Becattini, 1989; Brusco, 1982). La región sería, en este contexto, la estructura espacial espontánea definida por la aglomeración espacial de las pequeñas industrias integradas reticularmente con centros urbanos de diseño y gerencia de carácter global (Leborgne \& Lipietz, 1992). Esta primera observación, que estará en la base del imaginario regionalista e institucionalista hasta el presente, será cuestionada por Storper sobre la base de tres dificultades esenciales: 
En primer lugar, no hay en estos argumentos buenos motivos que expliquen por qué, en ciertas regiones, se observan proceso innovación, altos salarios y capacidades organizacionales mientras que en otras regiones ocurre exactamente lo contrario. En segundo lugar, no tiene herramientas para integrar en su cuadro de análisis formas de producción que no responden a estos patrones y que tienen plena vigencia, aún bajo el capitalismo informacional de producción flexible. Y, en tercer lugar y fundamentalmente, las observaciones no se apoyan en un "lenguaje analítico sobre la transformación industrial en curso" a nivel general (Storper, 1995: 197), lo que en buena medida explica las dos dificultades anteriores.

La crítica de Storper a esta primera versión institucionalista del problema regional, define con precisión el núcleo de la discusión: la observación de que estructuras institucionales inciden en el crecimiento de las regiones, pero se trata de estructuras institucionales sin una definición clara.

Si bien Storper presenta diferentes posiciones alternativas (como las del GREMI y los evolucionistas), conviene poner énfasis en el enfoque en el cual el propio autor se ubica. Este se apoyada en la noción de costos de transacción (Scott \& Storper, 1986), la cual tuvo absoluta centralidad en la renovación conceptual en la teoría marginalista desde fines de la década de 1960 (Medema, 2011).

Se repiten los términos del esquema analítico de Oliver Willamson acerca de los activos específicos administrados con mecanismos jerárquicos (Williamson, 1989) o, en este caso, con mecanismos colaborativos, lo que constituiría el principio de explicación de la aglomeración industrial partiendo de la base de los fallos al esquema marginalista puro. Sin embargo, ¿cómo emergen de la conducta utilitarista instituciones basadas en la jerarquía o la colaboración? Este problema es idéntico al que surge de la interpretación utilitarista de la cooperación, resumida en el contemporáneo trabajo de Robert Axelrod La evolución de la cooperación (1986). Storper reconoce los límites de esta lectura, no sólo al señalar que no todas las aglomeraciones urbanas son innovadoras sino, sobre todo, al observar la falta de una definición de los vínculos intersubjetivos que emergen de la proximidad.

Es importante notar el paralelo entre estos desarrollos y las proposiciones de la Nueva Geografía Económica: la pregunta de por qué ciertas las aglomeraciones innovan puede ser reescrita por ¿qué son las economías de aglomeración? Interrogante movilizado por Paul Krugman y sus colaboradores.

De un modo más general los marginalistas se encontraron ante la necesidad de hacer surgir el "espacio homogéneo e isótropo" (según la expresión de Aglietta y Orléan, 1990: 38), pliegues espacio temporales ligados a modos de comportamiento no previstos en el horizonte de la definición utilitarista del agente económico. Puede verse esto también en el propio movimiento interno de la economía espacial marginalista. Desde el modelo localizacional de Von Thünen la espacialidad había sido el resultado de accidentes geográficos tomados como datos externos y teorizados como costos de transporte (algo que puede deducirse de las conclusiones alcanzadas por Fujita, Krugman \& Venables, 2000: 26).

Esta pasividad y accidentalidad de las estructuras geográficas fue observada con sospecha particularmente por Alfred Marshall, quien vio en las aglomeraciones industriales una realidad económica que "flota en el aire", no reductible a los costos de transporte (Becattini, 2002). 
La Nueva Geografía Económica (NGE) se propuso romper el espacio homogéneo sin renunciar al modelo walrasiano de equilibrio general y, para ello, debieron introducir una distinción institucional: la hipótesis la competencia monopolística, como factor de explicación de la aglomeración industrial. No obstante, el resultado no fue lo suficientemente alentador: nuevamente los autores reconocieron el papel determinante de los datos iniciales (accidentalidad) y aun así, tuvieron que aceptar la existencia de múltiples equilibrios cuya selección dependerá de la "evolución" de soluciones anteriores (Brakman \& Garretsen, 2003).

Estos problemas espejados dieron el marco adecuado para que la escuela californiana y la NGE converjan en una solución común más allá de la fronteras marginalistas: observaron que las interacciones basadas en la proximidad geográfica, más específicamente en los encuentros cara a cara, sientan las bases de una modalidad de relaciones basadas en la confianza, lo que constituye el murmullo que da fortaleza a las ciudades (Storper \& Venables, 2004) convirtiéndose, a la postre, en activos relacionales (Storper, 1997) que explican el comportamiento innovador y las economías de aglomeración de un modo simultáneo.

Pero, este razonamiento, ¿no repite lo problemas que Storper observó en las diferentes perspectivas del debate regional? En primer lugar, las interacciones cara a cara y los actos comunicativos no son exclusivos de las aglomeraciones industriales exitosas. En segundo lugar, el pasaje de las interacciones cara a cara (de agentes utilitaristas), a la comunicación, de allí a relaciones de confianza y finalmente a la conversión en activos relacionales, es algo que simplemente no está desarrollado en esta perspectiva y no se derivan necesariamente de las premisas asumidas, al contrario, se oponen a ellas.

\section{La emergencia y definición del enfoque relacional: los sentidos de la proximidad}

La naturaleza institucionalizada de la acción económica se evidenció en este desarrollo de un modo patente. Y la supervivencia del modelo walrasiano de equilibrio general como situación normal reguladora perdió sentido con cada paso dado. La interpretación de las aglomeraciones innovadoras requerirá, por lo tanto, de una explicación institucional de fondo, es decir, en la que la aprehensión de la realidad económica sea el producto directo de una estructuración institucional.

Dos profundas renovaciones en el pensamiento científico constituyeron las bases filosóficas y epistemológicas del giro relacional de la geografía humana y económica, en torno al cual emergieron las teorías específicas de la proximidad, el territorio y la innovación. Por un lado, las teorías de los sistemas complejos y evolutivos que sacudieron las bases de las ciencias de la naturaleza a fines de la década de 1960 y comienzos de la de 1970 (Boulding, 1974; Morin, 1973; Stengers \& Prigogine, 1979; Bertalanffy, 1968). Por otro lado, contemporáneamente, el giro espacial de la teoría social y la crítica post estructuralista del sujeto (West-Pavlov -2009- resume este giro en tres figuras centrales, Kristeva, Foucault y Deleuze) los cuales desarticularon los pilares tanto humanistas como estructuralistas en la teoría social y abrieron el camino para cierta convergencia entre la tradición lingüística (fundamentalmente francesa) y pragmática (angloamericana). 
Estas dos renovaciones teóricas tuvieron unA influencia directa tanto en el campo de la geografía humana y económica como en las investigaciones sobre los sistemas de innovación, cuyo cruce y articulación definirá la base de los aportes relacionales analizados en este trabajo.

Simonsen (1996), tomando como antecedente el debate sintetizado por Gregory y Urry (1985), identifica a la noción de diferencia, como aquella que define a la nueva geografía humana del giro cultural. En este caso, la diferencia, la relación o la distinción, es una metáfora eminentemente espacial, por encima de los términos distinguidos. La relacionalidad constituye el ser social y por lo tanto coloca a la espacialidad como el fundamento de su aprehensión (Allen et al., 2012; Crampton \& Elden, 2007). Luego, si la sociedad es un sistema reticular de relaciones intersubjetivas, capaz de contener hábitos, instintos y reglas que no se definen a priori (porque de lo contrario se trataría de un sistema determinado por una sustancia trans-relacional), entonces se jerarquizan, en la investigación, las condiciones concretas y accidentales que son las que, efectivamente, pueden ser registradas por la observación. A esta conclusión llega Doreen Massey de forma muy temprana y será uno de las características centrales de este enfoque. En palabras de la autora: "The unique is back on the agenda" (Massey, 1985: 19).

La teoría del actor-red (Latour, 1996) es un ejemplo paradigmático de la influencia de estos movimientos. Allí, lingüística, pragmática y evolución se condensan en la red que constituye la estructura del estudio de la acción social, mientras que el grafo y espacio de coordenadas sociales, pasa a ser el lenguaje que formaliza esta metáfora. La conexión con los estudios relacionales de la proximidad y la innovación puede verse en la reconstrucción del concepto de embeddedness, donde el acto-red constituyó el núcleo de dicha interpretación (Granovetter, 1985) sentando las bases de la nueva sociología económica y finalmente utilizado para la conceptualización relacional del ambiente regional (Oinas, 1997).

La adopción de metáforas evolucionistas del cambio social (Boulding, 1981; Dosi et al., 1988; Nelson \& Winter, 1973) ha tenido la misma importancia en la formación de las principales hipótesis relacionales de la proximidad, el territorio y la innovación. El punto de contacto se abre en la posibilidad de combinar la indeterminación del enfoque relacional con las dos propiedades elementales de los sistemas evolutivos: complejidad y emergencia. La apertura del objeto relacional, su no determinación, permite combinar tanto la agregación de relaciones (complejidad) como la posibilidad de una relación que contenga el cambio de calidad. Para los estudios relacionales, el carácter dialógico de la cooperación concentrará esta potencia evolutiva y, a su vez, estará esencialmente conectada con la proximidad y la co-presencia en un espacio local.

Sobre la base de estas ideas, entre comienzos y mediados de la década de 1990, los estudios relacionales elaboraron diversas metáforas que expresaron la condensación en un mismo término del espacio geográfico local y las relaciones de cooperación. Los modelos de innovación territorial se multiplicaron: sistemas locales de innovación, regiones de aprendizaje, medios innovadores, entre otros (Moulaert \& Sekia, 2003).

Para el enfoque relacional, la proximidad pasaría a constituir el ámbito de las relaciones de cooperación / confianza / comunicación / comunidad pero no la razón suficiente de las mismas. El vínculo estructural entre la proximidad y los diferentes tipos de relaciones sociales, constituirá el núcleo problemático del enfoque relacional y este se manifestará en diferentes planos. 
En primer lugar, la influencia evidente que otros niveles del espacio social pueden tener al momento de determinar la especificidad de los sistemas de producción localizados. Esta consideración se observó tempranamente en las investigaciones vinculadas a la Teoría de la Regulación Francesa (Benko \& Lipietz, 1992), la cual convergerá con las premisas del modelo relacional.

En segundo lugar, el enfoque relacional debe definir de qué manera los vínculos de confianza, comunicación, cooperación, o comunidad se relacionan con la proximidad geográfica. Una pregunta que no tiene una respuesta evidente ni trivial. Y, finalmente, ello debe conciliarse con la observación generalizada de que en los sistemas productivos localizados subsisten grupos o agentes económicos con intereses divergentes, que tensionan con la realidad institucional de la cooperación.

Entre estas tres tensiones se le exigirá al enfoque relacional precisar el significado de las metáforas que supo construir y allí pondrá en juego su capacidad de dar adecuada respuesta a estos interrogantes.

Así, por ejemplo, Ash Amin y Frank Wilkinson (1999) buscaron precisar el sentido de la proximidad y exploraron las posibles relaciones entre dos modos genéricos de la proximidad: relacional y espacial. Blanc y Sierra (1999) abordaron este punto directamente: las formas organizacionales que facilitan la transmisión de conocimientos dependen no sólo de la proximidad espacial o geográfica, sino también de la proximidad cultural e institucional.

En ese mismo año, David Keeble y el propio Frank Wilkinson, se concentraron en el concepto de aprendizaje regional colectivo (Keeble \& Wilkinson, 1999: 295) y adoptaron una pregunta de base que puede extenderse a las ideas anteriores: ¿Cómo definir esa realidad social colectiva regional con la particular capacidad de aprender?.

Lawson y Lorenz mencionan, en diferentes momentos, a la reciprocidad y a la existencia de un lenguaje y una cultura común (Lawson \& Lorenz, 1999: 306-307) como los fundamentos de la cooperación que permite, por un lado, el manejo de los conocimientos tácitos que desbordan al mercado, pero también, por otro lado, la moderación y articulación de los conflictos intra firma y extra firma que supone el proceso de desarrollo.

Incluso los autores toman a John Commons ([1934] (1990) para encontrar pistas de una teoría social capaz de interpretar los sistemas económicos como un conjunto dinámico y articulado de diferentes grupos de intereses, que negocian en el marco de una unidad cultural común. Esta unidad es a la que permite un mínimo de reconocimiento mutuo, una disciplina transversal para quienes rompen las reglas elementales, y los límites de los daños recíprocos que pueden provocarse. Sin embargo, en el trabajo no se explicita, en ningún momento, la especificidad de los conflictos que atraviesan al sistema, así como tampoco, la naturaleza de ese plafón cultural común que permite su reproducción y da forma al proceso de formación y distribución del excedente.

Esta situación dejó el escenario adecuado para que Ann Markusen (1999) sacuda el avispero, marcando que los estudios regionales se apoyan en conceptos difusos que, paradójicamente, son los que tienen un papel clave en la explicación del desarrollo económico de las regiones. La autora observa el creciente uso de "una voz pasiva" (Markusen, 1999: 870) que funciona como 
actividad productora del proceso de desarrollo, pero que nunca es definida en sí y directamente. El Deux ex Machina de la innovación territorial.

A los pocos años de la publicación del trabajo de Markusen los estudios relacionales avanzaron en esfuerzos explícitos de precisión conceptual. Moulaert y Sekia (2003), construyeron un extenso mapa de diferentes "modelos de innovación territorial" y admiten la "ambigüedad y falta de claridad" (Moulaert \& Sekia, 2003: 299) en la definición de la territorialidad como factor activo del desarrollo innovativo. Sería preciso ampliar la mirada ontológica de la comunidad para alcanzar un modelo integrado de desarrollo que permita dar fundamento a los mecanismos no mercantiles de coordinación económica que, a su vez, se definen territorialmente (Moulaert \& Nussbaumer, 2005).

Lagendijk (2003), Peck (2003) y Hudson (2003), respondieron directamente a Markusen defendiendo la potencialidad de los estudios regionales (de base relacional), atentos precisamente al valor de la territorialidad (o alguna de sus modalidades) como concepto heurístico, llevando la discusión a un plano epistemológico y metodológico.

Peck (2003) argumenta contra la autora que la exploración de las relaciones sociales que rigen la institucionalización de la acción económica, no cae en el espectro de los criterios cuantitativos (una de las consecuencias de la crítica de Markusen). En línea con la argumentación de Peck, es posible es posible pensar que, si el objeto de estudio no es una cosa, sino una relación y su sentido intersubjetivo, este debe ser, fundamentalmente, comprendido (hermenéutica) y no sumado. Ello no impediría que, establecidos los significados de la acción económica, no puedan construirse modelos de análisis cuantitativos, es decir, en el marco de un modelo comprensivo en el que las cantidades tienen un significado específico.

Hudson (2003) lleva al límite las consecuencias del enfoque que él mismo denomina relacional. Según el autor, tanto la ciencia como la realidad es de naturaleza relacional y, por lo tanto, no tiene punto de apoyo ni forma de establecer pesos y medidas absolutos, sea a nivel de lo real como a nivel de su conocimiento. Este relativismo, intrínseco al pensamiento relacional, funcionaría, primero, como una justificación de la presencia de conceptos difusos y, segundo, favorecería la construcción de términos que se explican por sí mismos.

Sin embargo, la precisión del enfoque relacional sobre la proximidad, en tanto término "fashionable", encontrará su punto de partida en el trabajo de Torre y Gilly (2000: 169) quienes estudiaron las dimensiones analíticas de la proximidad y tuvieron un impacto significativo en el campo.

Según ellos la proximidad organizacional se apoya en formas institucionales basada en la similitud y la adhesión (pertenencia) (Torre \& Gilly, 2000: 174), abriendo un abanico de posibilidades institucionales de coordinación no mercantil que se despliegan en el espacio local-próximo muchas veces de forma no voluntaria.

Las "interacciones", tanto no intencionales como intencionales, apoyadas en la identificación y pertenencia entre personas, conformarían sistemas productivos en espacios geográficos acotados. Ellas tendrían un rol decisivo como explicación de las trayectorias innovativas. Las primeras como "externalidades tecnológicas, o según la atmósfera tecnológica de Marshall" (Torre \& Gilly, 
2000: 175), definiendo las condiciones de base para la adopción de una "trayectoria exitosa" (ídem). Entre "los esquemas de interacciones voluntarias que estructuran las estrategias de los agentes" los autores se enfocan en las "relaciones de co-operación, confidencia, intercambio de información técnica y el asociativismo", ello permite, en el marco de "densidad de interacciones" (ídem) mejorar la gestión del conocimiento y la eficiencia organizacional capaz de reducir costos de coordinación de los procesos de innovación.

La conexión entre territorio y proximidad se expone en el pleno sentido de la formulación relacional. Estas dos realidades, a la vez física y social, se conectan por una determinada definición institucional que, simultáneamente, fija territorialmente a la sociedad y explica su desempeño relativo, sobre la base de la unidad (similitud y adhesión), la complejidad (densidad) y la emergencia (co-operación).

\section{Los límites de la proximidad relacional: escalas y estructuración social}

Una vez analizado el núcleo conceptual del enfoque relacional, es posible profundizar en aquellas investigaciones que, sin abandonarlo, observaron limitaciones en las relaciones de cooperación locales como explicación del desempeño económico relativo de los sistemas localizados. En este apartado, se intentará mostrar cómo diferentes investigaciones convergieron sobre la reconsideración de la importancia relativa de escalas "no-locales" y las pujas de poder entre actores de un sistema productivo.

Oinas (2002) resume esta crítica al poner en cuestión, "la simple y popular conclusión: el éxito económico requiere clusterización" (Oinas, 2002: 65), tanto por la necesidad de una precisión conceptual así como también frente a la ingenuidad de las "happy communities". Lagendijk y Oinas, desarrollaron y sintetizaron esta idea sobre la base de que "las relaciones no locales tienen quizá la misma importancia que las locales para el éxito de las firmas y el ambiente regional" (Lagendijk \& Oinas, 2005: 11), a lo que agregaron, posteriormente, que la singularidad local, es el "producto del modo en que todo tipo de influencias, con diferentes articulaciones espaciales, se unen y mezclan localmente en formas particulares o 'geometrías del poder' (Massey, 1999)" (Lagendijk \& Oinas, 2005: 15).

La síntesis de Lagendijk y Oinas, se apoyó en un conjunto de artículos coordinado por Oinas y Asheim donde estos problemas teóricos fueron analizados. En dicho número Asheim intenta comprender las aglomeraciones industriales sobre la base del concepto de "spatial embeddedness" (Asheim, 2002: 112), que remite a la idea de una pluralidad de relaciones articuladas localmente que, sin embargo, pueden integrar diferentes escalas. El autor destaca los atributos típicos de los clústeres localizados, por ejemplo, "interacciones sociales, confianza e instituciones locales", que pueden ser definidos "más allá de los límites de la región", es decir, mediante relaciones con "sistemas de innovación no locales" con "efectos positivos sobre la trayectoria económica y la especialización industrial" (Asheim, 2002: 115).

Frente a esto el autor reconoce que se requieren enfoques para captar sistemas de innovación en multi nivel, y para ello se remite a la teoría de las cadenas globales de valor integrados 
en clusters locales (tomando como referencia a Cooke, 2001) como al enfoque de variedades de capitalismo, señalando la correspondencia de los sistemas regionales de innovación con las arquitecturas institucionales (nacionales) de las economías coordinadas (tomando como referencia los trabajos de Asheim y Herstad -2003- y Soskice -1999-) (Asheim, 2002: 119).

Finalmente, Asheim apela a una sugestiva formulación para la determinación de una trayectoria exitosa: las "coaliciones regionales desarrollistas" (Asheim, 2002: 121), que tendrían la particularidad de alinear los intereses de los agentes que operan en las tres escalas y potenciar la capacidad innovadora de la región, descubriendo allí el potencial de las relaciones de comunicación.

Tanto el problema de la multi escalaridad de los sistemas de innovación como de las relaciones de poder, fueron retomadas con mayor profundidad en los artículos de Bathelt y Taylor (2002) y Lagendijk (2002).

Bathelt y Taylor, por su parte, intentaron definir las relaciones de poder bajo una estricta lógica relacional. Para los autores, el poder se define "relacionalmente" ("as relationships") es decir, como asimetrías estructurales en la red de relaciones. Estas relaciones de poder producen "circuitos de poder" (Bathelt \& Taylor, 2002: 94) los cuales finalmente condensan en regímenes del producción y organización.

Bathelt y Taylor llegan a una conclusión algo paradójica sobre las relaciones de poder: los sistemas de producción tienen mayores probabilidades de éxito si logran estabilizar las luchas internas y establecer un estructura de jerarquías funcionales al crecimiento, pero al mismo tiempo, "demasiada jerarquía" (Bathelt \& Taylor, 2002: 104), puede conspirar contra el cambio tecnológico y la adaptabilidad del sistema, ya que una nueva tecnología puede amenazar posiciones dominantes. Lagendijk (2002) analiza directamente confluencia entre poder y escalas. El autor parte exactamente de esta observación: las escalas y el poder están relacionadas, pero ¿cómo?

En un plano, eminentemente relacional, el autor asume la distinción entre el mundo de la vida, es decir, el de las relaciones próximas de carácter dialógico, y el macrosistema de relaciones estructurales, como, por ejemplo, las relaciones de mercado y burocrático estatales (Lagendijk, 2002: 81), a los que denomina el sistema-mundo. Por otra parte, distingue la configuración geográfica de la vida, en la que rigen las distancias, y se observan comportamientos acotados localmente mientras que otros se extienden más allá de lo local, lo extra-local o no-local (Lagendijk, 2002: 84), según la denominación del autor.

Se observan aquí relaciones sociales de distinta naturaleza: las dialógicas/reflexivas (mundo de la vida) y las estructurales (sistema mundo), sin tener que atarlas a un espacio geográfico determinado. Ahora bien, si lo dialógico-reflexivo y lo estructural no coinciden con lo local y lo extra - local respectivamente, entonces ¿qué tipo de relaciones pueden preverse entre estas dos dimensiones de la vida social?

Lagendijk esboza una respuesta que, en líneas generales, podría considerarse características del enfoque relacional: primero, los agentes económicos podrían identificarse con la comunidad local, en tanto activo estratégico, para afrontar el proceso de desarrollo. Pero, ¿de dónde proviene esta comunidad? Lagendijk considera que la comunidad local puede ser pensada en los términos 
de la "perspectiva plural sobre las políticas de escala" (Lagendijk, 2002: 85-86), que nació precisamente como una perspectiva para estudiar las formas de la estatalidad en el post fordismo y los cambios en la espacialidad del Estado (Lagendijk, se apoya fundamentalmente en Brenner, 2001). Sobre la base de esta perspectiva Lagendijk resume el punto: "The central message in this work is: consider the regional scale, together with other scales, as being constructed through social and political processes" (Lagendijk, 2002: 85).

Sin embargo, es posible observar aquí, por un lado, cierta circularidad dado que, ¿no es preciso que exista alguna forma de comunidad para dar sustento al proceso político? o, alternativamente, cierta indeterminación, ya que el proceso político podría ser interpretado como una apertura irreductible a lo posible, en el marco de relaciones asimétricas cuyo contenido se define a posteriori, aun cuando se lo haga en diferentes escalas. En otros términos, Lagendijk logra captar la dificultad que supone definir la proximidad en sí misma y, por lo tanto, la necesidad de apelar a la articulación con otras escalas. Sin embargo, ello no le garantiza lograr una resolución del problema.

Por el contrario, Torre y Rallet (2005) explicitaron las lógicas de la proximidad "organizacional" (o relacional, según el modo en que se las viene nombrando en este trabajo), basada en la similitud y pertenencia (sutilmente diferentes entre sí) y observan con claridad que estas relaciones pueden ir más allá de lo local y que son, en definitiva, lo vínculos que sustentan modos de coordinación no mercantiles que generan ventajas económicas.

El vínculo entre la proximidad organizacional (o relacional según los términos seguidos en este trabajo) y geográfica tiende a perder protagonismo en este contexto. Torre y Rallet (2005) lo resaltan frente al avance de las tecnologías de la información y la comunicación, pero ello es sólo una razón aparente. Por el contrario, constituye una tendencia intrínseca a la definición de la proximidad organizacional:

"The spatial distribution of innovation activities appears to result from the varying ability of these mechanisms to deal with geographic distance. This is the core idea that lies at the intersection of geographical proximity and non-geographical proximity (organized proximity); it is non-geographical in that it can be defined as facilitating coordination in the absence of geographical proximity" (Torre \& Rallet, 2005: 430).

La desconexión entre la proximidad relacional y geográfica está, sustancialmente, rota. La territorialidad ya no tiene un lugar necesario, sino sólo accidental. Se trata de dos mundos ontológicamente separados cuya estructuración dependerá de condiciones no ponderables a priori.

Incluso Kevin Morgan (2004) advirtió sobre la "exagerada" muerte de la geografía en los estudios centrados en la proximidad relacional. El desliz es notable, el problema no sería tanto la muerte sino su muerte exagerada.

En cualquier caso, la conexión estructural entre un espacio relacional y el espacio geográfico dependerá de que el primero se defina mediante una relación social cuya forma contenga intrínsecamente una delimitación territorial o geográfica. Es el caso, por ejemplo, de la escala nacional, o el estado-nación soberano, con sus instituciones y sus medios de existencia, el cual encuentra 
en la delimitación territorial un atributo constitutivo. Morgan reconoce, en la conclusión de su trabajo, la importancia relativa del Estado nación para recuperar una teorización de la geografía e, incluso, observa su influencia en el desarrollo de las corporaciones globales o en el desempeño de los territorios o los sistemas territoriales de innovación (Morgan, 2004: 15)

Bouba-Olga y Grossetti, utilizan la expresión "proximité "socio-économique »" por oposición a la proximidad física, como una forma compleja y compuesta de lo que hasta aquí se denominó como proximidad relacional en un sentido amplio (Bouba-Olga \& Grossetti, 2008). En aquella categoría los autores identifican los siguientes componentes: "proximidad cognitiva", como formas de ver el mundo; "proximidad material", en relación con la posición de clase según la posesión de activos de distinta naturaleza. Ambas constituyen, una forma alternativa de converger sobre la idea de estructura social y los autores la combinan en el concepto de "proximidad de recursos". Sin embargo, la proximidad socioeconómica se complementa con otras dos modalidades de proximidad, la "proximidad relacional", según la posición del actor en la red de relaciones socioeconómicas (mercado, firma, cooperación), y la "proximidad de mediación", según la integración a dispositivos de integración comunicativa (lengua, medios de comunicación, redes sociales). Ambas convergen en la "proximidad de coordinación".

Poco después Bouba-Olga y Grossetti utilizan la expresión "proximité «socio-économique»" (Bouba-Olga \& Grossetti, 2008: 6-7), por oposición a la proximidad física, como una forma compleja y compuesta de lo que hasta aquí se denominó proximidad relacional en un sentido amplio. Los autores toman, por un lado, una noción proximidad (a la que llaman proximidad de recursos) de un modo comparable con el campo social en el sentido de Bourdieu (Bouba-Olga \& Grossetti, 2008: 8), es decir como una topología del espacio social según los recursos materiales o inmateriales de los agentes socio económicos. Por el otro, distinguen una noción de proximidad a la que llaman "de coordinación" (Bouba-Olga \& Grossetti, 2008: 9), definidas por los arreglos institucionales que articulan las relaciones entre los grupos. Allí distinguen la "proximidad relacional", según la posición del actor en la red de relaciones socioeconómicas (mercado, firma, cooperación), y la "proximidad de mediación", según la integración a dispositivos de integración comunicativa (lengua, medios de comunicación, redes sociales).

Al igual que la diferenciación escalar e, inmediatamente, las relaciones de poder, la formación y articulación de grupos sociales diferenciados, es decir, la estructuración social al interior del sistema emerge como dimensión ineludible para comprender el desempeño de las regiones. Frente a esto el modelo relacional (como pudo verse en el caso anterior) demostró que es capaz de incorporar todas estas dimensiones bajo la modalidad de una agregación y superposición de atributos que estructuran la proximidad. Sin embargo, debe notarse también que, en ningún caso, el espacio de relaciones, en un sentido amplio, logró establecer un vínculo estructural con el espacio geográfico, el cual se precipita sobre condiciones estrictamente accidentales. Al mismo tiempo, los modelos de proximidad relacional se multiplican y ponen en duda la posibilidad de lograr una lectura sistémica e integrada que permita definir criterios de inteligibilidad e interpretación de los sistemas y su desempeño.

En este sentido, por ejemplo, diferentes proyectos de síntesis avanzaron acumulando categorías convergentes. Podemos a penas mencionar Balland, Boschma y Frenken (2015), quienes buscaron establecer un modelo de "proximidad dinámica" convergente con las proposiciones de 
Bouba-Olga y Grossetti (2008). El mismo fue actualizado como co-evolución de redes de innovación articuladas (Broekel, 2015; Balland et al., 2020).

Finamente podemos mencionar la síntesis buscada por Torre y Wallet (2014) quienes agruparon a los principales autores de este enfoque con la mirada puesta de un "modelo integrado de proximidad" (Torre \& Wallet, 2014: 9) frente al "incremento en el número de categorías de la proximidad" (Torre \& Wallet, 2014: 20). Sin embargo, estos autores observaron la dificultad de lograr un único modelo coherente, oponiendo dos diferentes: las distinciones sobre la proximidad hechas por Boschma (cognitive proximity, organisational proximity, social proximity, institutional proximity) (Boschma, 2005) y las de Bouba-Olga y Grossetti analizadas arriba. Davids y Frenken (2018) también observaron esta dificultad y expresaron la necesidad de lograr un modelo integrado como la agenda a seguir por estas investigaciones.

\section{Proximidad relacional y la relación centro-periferia}

Es posible extender el razonamiento básico desarrollado en este trabajo a los resultados de las investigaciones que consideraron el problema de la relación centro-periferia desde el punto de vista relacional. En este apartado se intentará mostrar cómo las dificultades analizadas hasta aquí se repiten con cierta simetría cuando los modelos de proximidad relacional abordan la relación asimétrica entre diferentes sistemas. En este sentido, son numerosas las investigaciones que han observado que el destino de los ganadores está relacionado con el de los perdedores en el sistema mundo (Graffenberger \& Vonnahme, 2019; Shearmur, 2011), sin embargo, es preciso evaluar las hipótesis utilizadas para comprender las relaciones estructurales que ligan al centro con la periferia y los resultados alcanzados.

Jakob Eder realizó un exhaustivo trabajo de análisis bibliométrico, pero también conceptual y crítico, sobre la relación centro periferia en los estudios sobre proximidad e innovación. El autor presenta sus resultados sobre la base de dos criterios, el tipo de relaciones estudiadas y la definición la noción misma de periferia.

Sobre el primer punto observó investigaciones enfocadas en "los factores regionales", por ejemplo, políticas locales orientadas a la innovación, densidad institucional, participación de instituciones educativas, etc., los cuales mostrarían "una importancia limitada" (Eder, 2019: 126). Otras investigaciones se concentran en las redes globales que articulan nodos innovativos en la periferia vinculados con nodo que desde el centro planifican. Este abordaje reticular-global se pregunta por las posibilidades de articular estas actividades en sistemas regionales de innovación en la periferia. Eder observa resultados diversos que oscilan entre la "espesura" y la "fluidez", "institucional" y "organizacional" (Eder, 2019: 129). Finalmente, un tercer grupo de investigaciones concentradas en el reparto de tareas entre la periferia y el centro. En este caso, la periferia es observada como seguidora, mediante "innovación lenta", de la "innovación rápida" realizada en el centro (Eder, 2019: 130).

Como puede observarse las relaciones que cruzan a los espacios periféricos tienen diversa naturaleza e incluso distintas escalas. Podemos agregar aquí los resultados de investigaciones que, además, mostraron la relevancia de otras escalas en la trayectoria de las regiones periféricas 
incluso más allá de la presencia de factores regionales (Healy \& Morgan, 2012; Marques \& Morgan, 2020; Shearmur \& Bonnet, 2011).

Se produce aquí una suerte de bucle conceptual ya que definir una región periférica, bajo el lenguaje relacional, requiere una definición de la región y luego una definición la periferia, pero, sin embargo, ambos se implican. En este sentido, Eder encuentra que la mayor parte de los estudios analizados definen la periferia a partir de consideraciones físicas o demográficas (los costos de transporte y densidad poblacional) (Eder, 2019: 132) lo cual puede ser interpretado en función de la idea aquí desarrollada, es decir, la falta de un vínculo estructural entre el espacio relacional y el geográfico.

Lagendijk y Lorentzen, son un buen ejemplo en esta definición formal de la relación centro-periferia en relación con lo visto previamente: "la diferencia entre el centro y la periferia, es básicamente una diferencia de poder" (Lagendijk \& Lorentzen, 2007: 462), en un sentido lo suficientemente genérico y abstracto, como para representar un amplio espectro de situaciones. Las cadenas globales, los espacios nacionales y regionales, se articulan bajo el lenguaje de los campos de fuerza en el que la posición de un espacio local supone una combinación de condiciones particulares, tecnológicas, geográficas y relacionales, que definen su situación central o periférica. El recorte local es, en este contexto, mas o menos arbitrario y, en todo caso, se condensa en la idea de que la proximidad organizacional (cognitiva e institucional) mantiene cierto vínculo con el espacio local. La base local de la proximidad organizacional es la que permitiría mejorar la posición del sistema en la cadena global, aunque, "in the end, both societal and firm level resources are needed to enable the mobility which is required to 'conquer' physical and other forms of distance" (Lagendijk \& Lorentzen, 2007: 466).

Sin embargo, es probable que la formulación más radicalizada de la relación centro periferia bajo el lenguaje relacional se encuentre en la idea de "modelos de perifericidad aespacial" según la expresión de Andrew Copus (2001: 547). En este caso, la distinción entre lo relacional y lo geográfico se lleva al límite el carácter formal del enfoque convirtiendo al grafo y a las nodalidades asimétricas en la estructura básica del sistema sin espacialidad geográfica inherente.

En este sentido, la posición periférica (o central) es, esencialmente, una posición en la red de relaciones (actor-red o nodo de actores-red) mientras que un espacio geográfico o territorio periférico, dependerá de la fijación de dicha red al espacio geográfico, sin que pueda establecerse una correspondencia necesaria entre ambos planos.

En resumen, la naturaleza formal y abstracta del modelo relacional, no permite establecer relaciones necesarias o estructurales entre el espacio relacional (con su estructura formal definida por nodos centrales y periféricos) y el espacio geográfico. En definitiva, las relaciones centro periferias no son producidas por la articulación entre las relaciones sociales de producción y sus especificidades histórico estructural, sino postuladas, directa o indirectamente, en el marco del espacio relacional. Esta postulación hace que los factores determinantes de la estructura centro periferia, tal y como ocurría con el caso del desempeño económico relativo, se incorporen como condiciones contextuales o accidentes geofísicos o estrategias de poder a partir de un juego de posiciones estratégicas en el mismo campo que se quiere definir, o una combinación de todas estas. 
Así, por ejemplo, además de los trabajos ya mencionados, son muchos los que reconocen al Estado Nacional como una institución significativa al momento de incidir en las trayectorias de especialización productiva y tecnológica (Július \& Richard, 1999; Morgan et al., 2017; Pontarollo \& Serpieri, 2020). O incluso de un modo más amplio, son los esquemas de gobernanza multi nivel los que rigen las condiciones necesarias para comprender la formación de centralidades y periferias, donde se pone en juego la planificación misma de los sistemas regionales (Elcock -2003desarrolla precisamente esta idea aplicada al caso de la centralidades y periferias producidas en el Reino Unidos).

Sin embargo, frente a ello, el enfoque relacional, que parte de un modelo marcado por la dualidad entre un lenguaje abstracto (que admite la complejidad, la asimetría y la emergencia) sólo podrá conectarse con las condiciones físicas y materiales mediante suposiciones institucionales ad hoc. Sólo así puede fijar la proximidad organizacional al espacio local y postular estructuras de gobernanza (o alianzas de grupos) que logran imponer sus estrategias en el campo social.

Incluso, estos enfoques han llegado a idealizar un modelo de proximidad local y desarrollo de la periferia. Según el mismo, actores-red periféricos radicados en un espacio local, sobre la base de la reflexividad y la identificación, pueden lograr ganar grados de libertad en la planificación de sus actividades (de innovación) integradas o conectadas a la planificación desarrollada en nodos centrales y, por lo tanto, incrementar sus niveles de acumulación de capital e infraestructura (Berman et al., 2020; Copus et al., 2008; Fitjar \& Rodríguez-Pose, 2011; Grillitsch \& Nilsson, 2017). Este es, desde luego, un caso posible, incluso relevante, aunque, nuevamente, resulta un caso extremadamente particular (no generalizable) e incluso no necesariamente probable (así lo indican por ejemplo, Godin \& Vinck, 2017 y Oughton et al., 2002), donde la especificidad de las relaciones sociales (históricas y estructurales) que producen las distintas formas de territorialidad no tiene un reconocimiento explícito.

\section{A modo de conclusión: síntesis y el camino a una agenda alternativa}

Desde las primeras intuiciones institucionalistas sobre la atmósfera de las aglomeraciones industriales, se desarrolló un debate entre diferentes programas de investigación sobre la naturaleza de los entornos institucionales en los que se producen los procesos de acumulación de capital y desarrollo tecnológico con sus patrones territoriales.

Para el análisis de este debate se tomó como hipótesis de lectura la oposición entre los supuestos básicos de dos teorías socio económicas alternativas: el marginalismo y el enfoque relacional. Se intentó argumentar que esta distinción remite a dos fundamentaciones opuestas de la relación espacio-sociedad: fundamento utilitarista y el modelo relacional de la acción social.

El primero de los modelos evolucionó para dar cuenta de las interacciones cara a cara y los actos comunicativos, utilizándolos como fundamentos del desempeño económico relativo. Sin embargo, no pudo resolver cómo, entre agentes utilitarias, nacen interacciones cara a cara que dan lugar a la comunicación, de allí a relaciones de confianza y finalmente a la conversión en activos 
relacionales. Estas modalidades de la interacción social no se derivan necesariamente de las premisas asumidas (utilitarismo como fundamento de la conducta), al contrario, se oponen a ellas.

El modelo relacional, por su parte, se impuso aportando un lenguaje para captar la diversidad institucional que el enfoque marginalista (cerrado y a apriorístico) clausuraba, y dio lugar a las nociones de sistema relacional, complejidad y dinámica evolutiva. Más aun, pudo distinguir con claridad la realidad intersubjetiva relacional de la estrictamente física o geográfica, dando privilegio a la primera como objeto de la teoría social.

Sin embargo, asumir esta dualidad tuvo como consecuencia el quiebre de los nexos necesarios entre ambos momentos, de modo tal que toda fijación territorial del espacio relacional implicaba una serie de hipótesis institucionales apoyadas en la arbitrariedad. Esta limitación se hizo presente en una serie concatenada de dificultades que emergieron ante la necesidad de dar cuenta de los procesos reales de formación sistemas productivos territorializados: la falta de precisión conceptual de la proximidad relacional, el reconocimiento de la multiescalaridad de los fenómenos estudiados, la diferenciación estructuración conflictiva entre clases o grupos y, finalmente, la estructuración espacial de espacios centrales y periféricos.

Cada una de estas problemáticas fueron llevando al enfoque relacional a la adición de capas o dimensiones sin que ello pueda echar luz sobre la articulación entre las mismas, poniendo en evidencia con mayor profundidad la necesidad de una reconsideración de los supuestos más profundos con los que aborda la realidad socioeconómica. La estructuración territorial de los sistemas de producción permanecerá en la accidentalidad mientras su abordaje no provenga de la explicitación de las relaciones sociales de producción que, en su especificidad histórico-estructural, la producen.

Poner el comienzo en las relaciones sociales histórico-estructurales, conduce a jerarquizar el momento en el que éstas se articulan, conflictiva y complementariamente, y producen allí una realidad socio-espacial en diferentes escalas: global-capitalista, nacional-estatal, local-comunitaria, y en las que diferentes grupos o clases se identifican entre sí al mismo tiempo que entran en conflicto. Otorgar inteligibilidad a la unidad (es decir, la raíz histórica común que permite conciliar las diferentes relaciones y sus respectivas escalas) y a la diferenciación (es decir, la especificación de diferentes escalas y clases o grupos) constituye la meta general de esta agenda de investigación.

Trabajos como los Lefebvre (1974), Wallerstein (1998) o Arrighi y Drangel (1986), constituyen antecedentes directos de estos objetivos, pero sigue pendiente la terea de construir programas de investigación que puedan establecer modos de caracterización de los sistemas productivos localizados en este marco. A esto debe agregarse la dificultad de pensar la especificidad de la periferia, y la oportunidad que esta agenda abre a la reinterpretación y actualización de las tesis centrales del estructuralismo latinoamericano que indagaron la condición periférica como producto de la estructura social, y la articulación de los sistemas nacionales con la economía global. Estos antecedentes constituyen un verdadero aliciente para una reformulación del análisis de la proximidad socioeconómica y geográfica y los procesos de desarrollo relativo con la posibilidad de lograr un análisis integral incorporando las dimensiones ausentes analizadas. 


\section{Referencias}

AGLIETTA, M., \& ORLÉAN, A. La violencia de la moneda. Buenos Aires: Siglo XXI, 1990.

ALLEN, J., CHARLESWORTH, J., COCHRANE, A., COURT, G., HENRY, N., MASSEY, D., \& SARRE, P. Rethinking the Region: Spaces of Neo-Liberalism. London: Routledge, 2012. https://doi. org/10.4324/9780203007501.

ARRIGHI, G., \& DRANGEL, J. The stratification of the world-economy: an exploration of the semiperipheral zone. Review (Fernand Braudel Center), 1986, Vol. 10, No 1, p. 9-74.

ASHEIM, B. T. Temporary organisations and spatial embeddedness of learning and knowledge creation. Geografiska Annaler: Series B, Human Geography, 2002, Vol. 84, No 2, p. 111-124. https:// doi.org/10.1111/j.0435-3684.2002.00117.x

ASHEIM, B., \& HERSTAD, S. Regional clusters under international duress: Between local learning and global corporations. En: ASHEIM, B. T. \& MARIUSSEN, Å. (eds.) Innovations, Regions and Projects: Studies in new forms of knowledge governance. Stockholm: Nordregio, 2003, p. 203-239.

AXELROD, R. La evolución de la cooperación: El dilema del prisionero y la teoría de los juegos. Madrid: Alianza, 1986.

BALLAND, P.-A., BOSCHMA, R., \& FRENKEN, K. Proximity and Innovation: From Statics to Dynamics. Regional Studies, 2015, Vol. 49, N6, p. 907-920. https://doi.org/10.1080/00343404.2014.883598

BALLAND, P.-A., BOSCHMA, R., \& FRENKEN, K. Proximity, Innovation and Networks: A Concise Review and Some Next Steps. Papers in Evolutionary Economic Geography PEEG, 2020. https:// ideas.repec.org/p/egu/wpaper/2019.html

BATHELT, H., \& TAYLOR, M. Clusters, power and place: Inequality and local growth in time-space. Geografiska Annaler: Series B, Human Geography, 2002, Vol. 84, No2, p. 93-109. https://doi. org/10.1111/j.0435-3684.2002.00116.x

BECATTINI, G. Del distrito industrial marshalliano a la «teoría del distrito» contemporánea. Una breve reconstrucción crítica. Investigaciones Regionales-Journal of Regional Research, 2002, N01, p. 9-32.

BECATTINI, G. Riflessioni sul distretto industriale marshalliano come concetto socio-economico. Stato e mercato, 1989, p. 111-128.

BENKO, G., \& LIPIETZ, A. Les régions qui gagnent: Districts et réseaux: les nouveaux paradigmes de la géographie économique. Paris : Presses universitaires de France, 1992.

BERMAN, A., MARINO, A., \& MUDAMBI, R. The global connectivity of regional innovation systems in Italy: A core-periphery perspective. Regional Studies, 2020, Vol. 54, N05, p. 677-691. https://doi. org/10.1080/00343404.2019.1672865 
BERTALANFFY, L. General system theory: Foundations, Development, Applications. New York: George Braziller, 1968.

BLANC, H. \& SIERRA, C. The internationalisation of R\&D by multinationals: A trade-off between external and internal proximity. Cambridge Journal of Economics, 1999, Vol. 23, No2, p. 187-206. https://doi.org/10.1093/cje/23.2.187

BOSCHMA, R. Proximity and Innovation: A Critical Assessment. Regional Studies, 2005, Vol. 39, N01, p. 61-74. https://doi.org/10.1080/0034340052000320887

BOUBA-OLGA, O., \& GROSSETTI, M. Socio-économie de proximité. Revue dEconomie Regionale Urbaine, 2008, octobre 3, p. 311-328.

BOULDING, K. E. Evolutionary Economics. SAGE Publications, 1981.

BOULDING, K. E. Toward a general social science. Colorado Associated University Press, 1974.

BRAKMAN, S., \& GARRETSEN, H. Rethinking the "New' Geographical Economics. Regional Studies, 2003, Vol. 37, N6-7, p. 637-648. https://doi.org/10.1080/0034340032000108732

BROEKEL, T. The Co-evolution of Proximities - A Network Level Study. Regional Studies, 2015, Vol. 49, N6, p. 921-935. https://doi.org/10.1080/00343404.2014.1001732

BRUSCO, S. Small firms and industrial districts: the experience of Italy. En: KEEBLE, D. \& WEVER, E. New firms and regional development in Europe. London and New York: Croom Helm, 1986, p. 184-202.

COOKE, P. Regional Innovation Systems, Clusters, and the Knowledge Economy. Industrial and Corporate Change, 2001, Vol. 10, No4, p. 945-974. https://doi.org/10.1093/icc/10.4.945

COPUS, A. K. From Core-periphery to Polycentric Development: Concepts of Spatial and Aspatial Peripherality. European Planning Studies, 2001, Vol. 9, No4, p. 539-552. https://doi. org/10.1080/713666491

COPUS, A., SKURAS, D., \& TSEGENIDI, K. Innovation and Peripherality: An Empirical Comparative Study of SMEs in Six European Union Member Countries. Economic Geography, 2008, Vol. 84, N01, p. 51-82. https://doi.org/10.1111/j.1944-8287.2008.tb00391.x

CRAMPTON, J. W., \& ELDEN, S. Space, Knowledge and Power: Foucault and Geography. Ashgate Publishing, 2007.

DAVIDS, M., \& FRENKEN, K. Proximity, knowledge base and the innovation process: Towards an integrated framework. Regional Studies, 2018, Vol. 52, N01, p. 23-34. https://doi.org/10.1080/003 43404.2017.1287349 
DOSI, G., FREEMAN, C., NELSON, R., SILVERBERG, G., \& SOETE, L. (eds). Technical Change and Economic Theory. London and New York: Printer Publisher, 1988.

EDER, J. Innovation in the Periphery: A Critical Survey and Research Agenda. International Regional Science Review, 2019, Vol. 42, No2, p. 119-146. https://doi.org/10.1177/0160017618764279

ELCOCK, H. Networks, Centres and Peripheries: Strategic Planning in a European State. Regional \& Federal Studies, 2003, Vol. 13, No3, p. 44-65. https://doi.org/10.1080/13597560308559434

FITJAR, R. D., \& RODRÍGUEZ-POSE, A. Innovating in the Periphery: Firms, Values and Innovation in Southwest Norway. European Planning Studies, 2011, Vol. 19, N4, p. 555-574. https://doi.org/10.1 080/09654313.2011.548467

FUJITA, M., KRUGMAN, P. \& VENABLES, A. J. Economía espacial: las ciudades, las regiones y el comercio internacional, Ariel, 2000.

GODIN, B., \& VINCK, D. Introduction: Innovation - from the forbidden to a cliché. En: GODIN, B., \& VINCK, D. (eds) Critical Studies of Innovation. Alternative Approaches to the Pro-Innovation Bias. Edward Elgar, 2017, p. 1-14.

GRAFFENBERGER, M., \& VONNAHME, L. Questioning the "periphery label» in economic geography: Entrepreneurial Action and Innovation in South Estonia. ACME: An International Journal for Critical Geographies, 2019, Vol. 18, p. 529-550.

GRANOVETTER, M. Economic action and social structure: The problem of embeddedness. American journal of sociology, 1985, Vol. 91, N³, p. 481-510. https://doi.org/10.1086/228311

GREGORY, D. y URRY, J. (Eds.). Social relations and spatial structures, Palgrave, 1985.

GRILLITSCH, M., \& NILSSON, M. Firm performance in the periphery: On the relation between firm-internal knowledge and local knowledge spillovers. Regional Studies, 2017, Vol. 51, No8, p. 1219-1231. https://doi.org/10.1080/00343404.2016.1175554

HEALY, A., \& MORGAN, K. Spaces of Innovation: Learning, Proximity and the Ecological Turn. Regional Studies, 2012, Vol. 46, No8, p. 1041-1053. https://doi.org/10.1080/00343404.2012.672725

HUDSON, R. Fuzzy Concepts and Sloppy Thinking: Reflections on Recent Developments in Critical Regional Studies. Regional Studies, 2003, Vol. 37, N6-7, p. 741-746. https://doi. org/10.1080/0034340032000108822

JÚLIUS, P. H., \& RICHARD, P. G. Core and Periphery in the World Economy: An Empirical Assessment of the Integration of the Developing Countries Into the World Economy. International Economic Journal, 1999, Vol. 13, N4, p. 35-51. https://doi.org/10.1080/10168739900000043 
KEEBLE, D., \& WILKINSON, F. Collective Learning and Knowledge Development in the Evolution of Regional Clusters of High Technology SMEs in Europe. Regional Studies, 1999, Vol. 33, No4, p. 295-303. https://doi.org/10.1080/00343409950081167

LAGENDIJK, A. \& OINAS, P. Proximity, external relations, and local economic development. En: LAGENDIJK, A. \& OINAS, P. (Eds) Proximity, distance and diversity, issues on economic interaction and local development, Routledge, 2005, p. 3-22.

LAGENDIJK, A. Beyond the regional lifeworld against the global systemworld: Towards a relational -scalar perspective on spatial-economic development. Geografiska Annaler: Series B, Human Geography, 2002, Vol. 84, No2, p. 77-92. https://doi.org/10.1111/j.0435-3684.2002.00115.x

LAGENDIJK, A. Towards Conceptual Quality in Regional Studies: The Need for Subtle Critique - A Response to Markusen. Regional Studies, 2003, Vol. 37, No6-7, p. 719-727. https://doi. org/10.1080/0034340032000108804

LAGENDIJK, A., \& LORENTZEN, A. Proximity, Knowledge and Innovation in Peripheral Regions. On the Intersection between Geographical and Organizational Proximity. European Planning Studies, 2007, Vol. 15, N4, p. 457-466. https://doi.org/10.1080/09654310601133260

LATOUR, B. On actor-network theory: A few clarifications. Soziale Welt, 1996, Vol. 47, No4, p. 369 381.

LAWSON, C., \& LORENZ, E. Collective Learning, Tacit Knowledge and Regional Innovative Capacity. Regional Studies, 1999, Vol. 33, No4, p. 305-317. https://doi.org/10.1080/713693555

LEBORGNE, D., \& LIPIETZ, A. Idées fausses et questions ouvertes de l'après-fordisme. Espaces et sociétés, 1992, Vol.1, p. 39-68.

LEFEBVRE, H. La production de l'espace. Éditions Anthropos, 1974.

MARKUSEN, A. Fuzzy Concepts, Scanty Evidence, Policy Distance: The Case for Rigour and Policy Relevance in Critical Regional Studies. Regional Studies, 1999, Vol. 33, No9, p. 869-884. https:// doi.org/10.1080/00343409950075506

MARQUES, P., \& MORGAN, K. Innovation without regional development? The complex interplay of innovation, institutions and development, Papers in Innovation Studies, 2020, No3. Pre-print

MASSEY, D. New directions in space. En: GREGORY, D. y URRY, J. (Eds.). Social relations and spatial structures, Palgrave, 1985, p. 20-48.

MEDEMA, S. G. A case of mistaken identity: George Stigler, "The Problem of Social Cost," and the Coase theorem. European Journal of Law and Economics, 2011, Vol. 31, No1, p. 11-38. https://doi. org/10.1007/s10657-010-9196-5 
MORGAN, K. The exaggerated death of geography: learning, proximity and territorial innovation systems. Journal of Economic Geography, 2004, Vol. 4, No1, p. 32-49. https://doi.org/10.1093/ jeg/4.1.3

MORGAN, K., MUNDAY, M., \& ROBERTS, A. Local economic development opportunities from NHS spending: Evidence from Wales. Urban Studies, 2017, Vol. 54, N013, p. 3138-3156. https://doi. org/10.1177/0042098016658248

MORIN, E. Le paradigme perdu: La nature humaine. Le Seuil,1973.

MOULAERT, F. \& NUSSBAUMER, J. Defining the Social Economy and its Governance at the Neighbourhood Level: A Methodological Reflection. Urban Studies, 2005, Vol. 42, N011, p. 2071-2088. https://doi.org/10.1080/420980500279752

MOULAERT, F. \& SEKIA, F. Territorial Innovation Models: A Critical Survey. Regional Studies, 2003, Vol. 37, No3, p. 289-302. https://doi.org/10.1080/0034340032000065442

NELSON, R. R., \& WINTER, S. G. Toward an Evolutionary Theory of Economic Capabilities. The American Economic Review, 1973, Vol. 63, No2, p. 440-449.

OINAS, P. Competition and collaboration in interconnected places: Towards a research agenda. Geografiska Annaler: Series B, Human Geography, 2002, Vol. 84, No2, p. 65-76. https://doi. org/10.1111/j.0435-3684.2002.00114.x

OINAS, P. On the Socio-Spatial Embeddedness of Business Firms, Erdkunde, 1997, Vol. 51, No1, p. 23-32. https://doi.org/10.3112/erdkunde.1997.01.03

OUGHTON, C., LANDABASO, M., \& MORGAN, K. The Regional Innovation Paradox: Innovation Policy and Industrial Policy. The Journal of Technology Transfer, 2002, Vol. 27, N01, p. 97-110. https:// doi.org/10.1023/A:1013104805703

PECK, J. Fuzzy Old World: A Response to Markusen. Regional Studies, 2003, Vol. 37, No6-7, p. 729 740. https://doi.org/10.1080/0034340032000108813

PIORE, M. J., \& SABEL, C. F. The second industrial divide: Possibilities for prosperity. Basic Books, 1986.

PONTAROLLO, N., \& SERPIERI, C. Towards regional renewal: A multilevel perspective for the EU. Regional Studies, 2020, Vol. 54, No6, p. 754-764. https://doi.org/10.1080/00343404.2019.1640357

SCOTT, A. J., \& STORPER, M. Production, work, territory the geographical anatomy of industrial capitalism. London: Allen and Unwin, 1986.

SHEARMUR, R. Innovation, regions and proximity: From neo-regionalism to spatial analysis. Regional Studies, 2011, Vol. 45, No9, p. 1225-1243. https://doi.org/10.1080/00343404.2010.484416 
SHEARMUR, R., \& BONNET, N. Does local technological innovation lead to local development? A policy perspective. Regional Science Policy \& Practice, 2011, Vol. 3, No3, p. 249-270. https://doi. org/10.1111/j.1757-7802.2011.01040.x

SIMONSEN, K. What kind of space in what kind of social theory? Progress in Human Geography, 1996, Vol. 20, No 4, p. 494-512. https://doi.org/10.1177/030913259602000404

SOSKICE, D. Divergent production regimes: Coordinated and uncoordinated market economies in the 1980s and 1990s. En: H. KITSCHELT, P. LANGE, G. MARKS, \& J. D. STEPHENS (eds), Continuity and Change in Contempary Capitalism, Cambridge University Press 1999, pp. 101-134.

STENGERS, I., \& PRIGOGINE, I. La nouvelle alliance: Métamorphose de la science. Gallimard 1979.

STORPER, M. The resurgence of regional economies, ten years later: The region as a nexus of untraded interdependencies. European urban and regional studies, 1995, Vol. 2, No3, p. 191-221. https://doi.org/10.1177/096977649500200301

STORPER, M. Regional economies as relational assets. En: R. LEE, \& J. WILLS, J. (eds.), Geographies of Economies, London: Edward Arnold, 1997, p. 248-258.

STORPER, M., \& VENABLES, A. J. Buzz: Face-to-face contact and the urban economy. Journal of economic geography, 2004, Vol. 4, N4, p. 351-370. https://doi.org/10.1093/jnlecg/lbh027

TORRE, A., \& GILLY, J.-P. On the Analytical Dimension of Proximity Dynamics. Regional Studies, 2000, Vol. 34, No2, p. 169-180. https://doi.org/10.1080/00343400050006087

TORRE, A., \& RALLET, A. Proximity and Localization. Regional Studies, 2005, Vol. 39, N01, p. 47-59. https://doi.org/10.1080/0034340052000320842

TORRE, A., \& WALLET, F. Regional Development and Proximity Relations. Edward Elgar Publishing. 2014.

WALLERSTEIN, I. (1998). The time of space and the space of time: The future of social science. Political geography, 1998, Vol. 17, No1, p. 71-82. https://doi.org/10.1016/S0962-6298(96)00097-2

WEST-PAVLOV, R. Space in Theory: Kristeva, Foucault, Deleuze. Rodopi, 2009.

WILLIAMSON, O. E. Transaction cost economics. En: SCHMALENSEE, R. AND WILLIG, R. (Eds) Handbook of industrial organization, Elsevier, 1989, pp. 135-182. 